DEPARTMENT OF COMMERCE

BUREAU OF STANDARDS

S. W. STRATTON, Director

SCIENTIFIC PAPERS OF THE BUREAU OF STANDARDS, No. 333

[Issued March 20, 1919]

\title{
OPTICAL CONDITIONS ACCOMPANYING THE STRIAE WHICH APPEAR AS IMPERFECTIONS IN OPTICAL GLASS
}

By Lieut. Commander A. A. Michelson, U. S. N., R. F.

Striae in optical glass may conveniently be divided into two classes: First, those which appear under proper conditions as isolated bright streaks on a dark background and, second, those in which such bright streaks are very numerous and run into one another, forming bright but irregularly continuous bands.

Even a superficial examination of the two kinds shows that the optical qualities of the former type are practically unaffected by the striae, while the latter specimens are in general unfitted for use in optical instruments.

For the investigation of the former class a specimen containing a single approximately plane and very narrow stria-resembling a crack in the glass-was ground and polished so that the stria was approximately perpendicular to the polished plane parallel surfaces.

This specimen shows "total reflection" at the stria when the "grazing" angle is sufficiently small (from zero to about 20 degrees) and equally on either surface of the stria.

At greater angles there is an abrupt drop in the intensity to nearly zero.

If it be admitted that these peculiarities are caused by a layer of smaller index of refraction than the remaining portion of the specimen, the maximum change in index may be readily calculated from the "grazing angle." This was found to be from $3^{\circ}$ to $6^{\circ}$ for this specimen (corresponding to an exterior angle of $4.5^{\circ}$ to $9^{\circ}$ ). From this it follows that the ratio of indices is approximately from 0.995 to 0.9975 , or the difference in refractive index at the stria is from 0.005 to 0.0025 .

(It may be noted that if the stria was in reality a crack, which it so strongly resembles, the exterior grazing angle should be so $92112^{\circ}-19$ 
large that total reflection under the conditions of the experiment would occur at all incidences.)

Again if the intensities be plotted with $\frac{\partial n}{n}=-0.005$ and $\frac{\partial n}{n}=+0.005$, the corresponding graphs arawn in the figure show

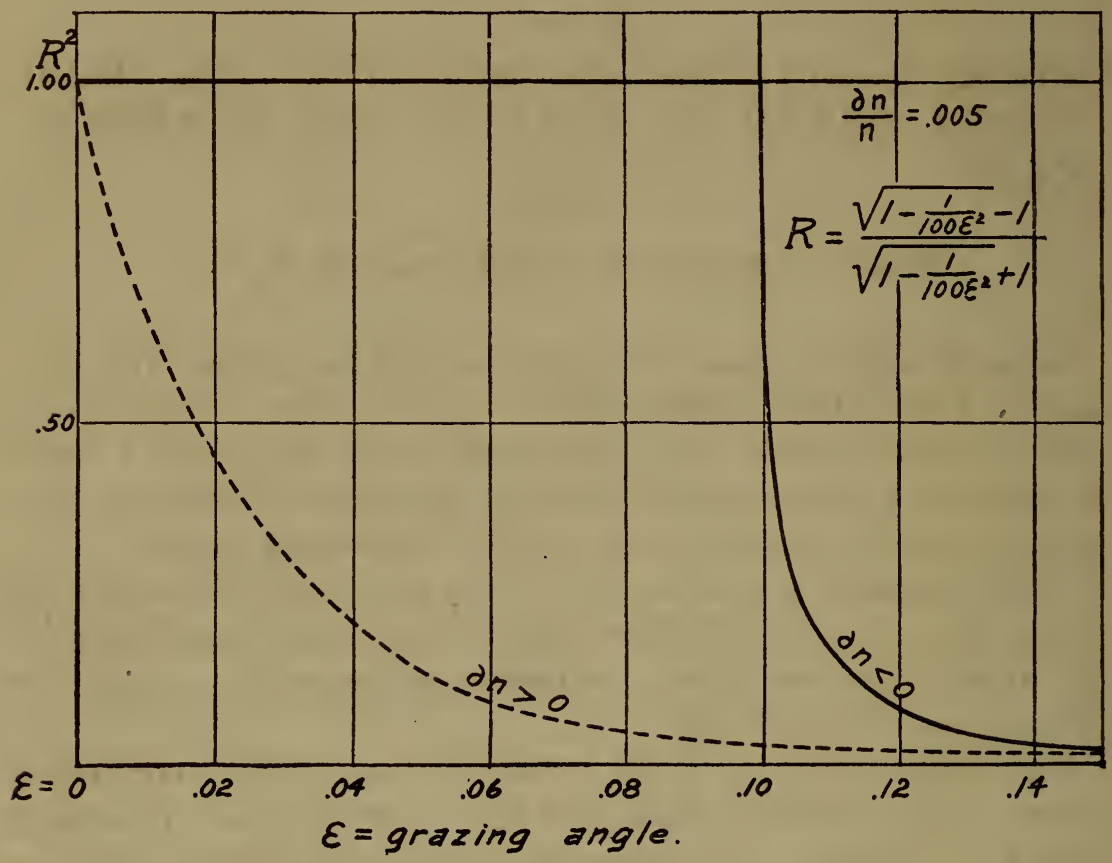

FIG. I

in the former case (smaller index at stria) $\delta$ the sudden drop in intensity at "grazing angle" $\epsilon=0 . \mathrm{I}$, while in the other case (larger index at stria) there is a steady diminution from $\epsilon=0$ to $\epsilon=90^{\circ}$.

This shows conclusively that this stria is caused by a film of smaller index of refraction than the remainder of the glass.

It was hoped that the following experiment would give the law of change of index as the stria is approached from either side:

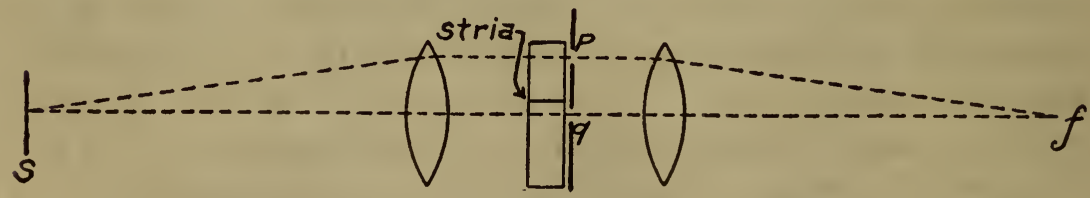

FIG. 2

The image of a tungsten filament was concentrated on the slit $s$ of a collimator, and the parallel rays fell on the specimen. Thence two pencils passed through the apertures $p$ and $q$ in a screen to the 
objective of the observing telescope at the focus of which at $f$, interference fringes are observed. The position of these was noted as the aperture $q$ was moved past the stria, and careful observation showed no appreciable movement of the fringes until the aperture was exactly over the stria, when the fringes disappeared.

A motion of one-tenth the distance between the fringes is clearly measurable, and with a thickness of the specimen corresponding to 16000 light waves it follows from the formula

$$
\begin{aligned}
& \partial N=\frac{t}{\lambda}\left(n_{1}-n_{2}\right)<0 . \mathrm{I} \\
& n_{1}-n_{2}<\frac{\mathrm{I}}{\mathrm{I} 60000}
\end{aligned}
$$

which shows that, within the limit of errors of observation, there is no change in index of refraction until the stria itself is reached.

The thickness of the stria was found to be something less than a fourth of a millimeter. In order to get a somewhat more accurate estimate, however, another specimen was cut with a face inclined at an angle of about $1 / 30$ to the plane of the stria. The total reflection at $K$ gave an edge whose width was of the order

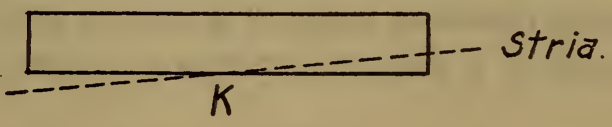

FIG. 3

of $\mathrm{I} / 4 \mathrm{~mm}$ or less, so that the corresponding width of the stria must have been less than $\mathrm{I} / \mathrm{r} 20 \mathrm{~mm}$.

A still more convincing confirmation of the preceding results is furnished by the following interferometer test:

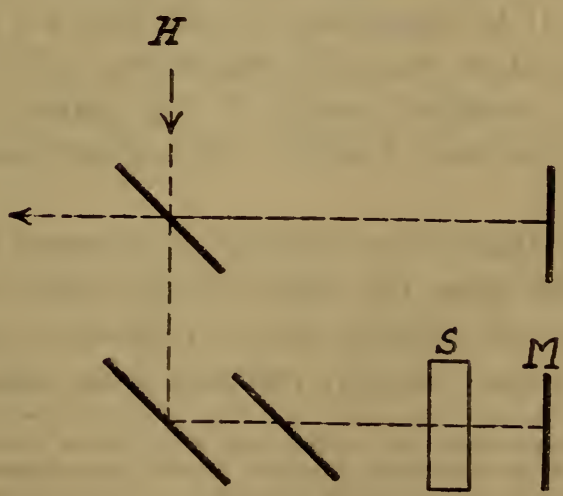

FIG. 4 
The interferometer arranged as shown diagrammatically in the figure receives light from a helium tube $H$, which traverses in one of the branches the specimen $S$, containing the vertical stria.

The interference fringes are arranged to appear as horizontal bands coinciding with the surface of the mirror $M$. These appear entirely straight, except at the stria, where they merge abruptly into the narrow dark line which is formed by the projection of the stria.

If the specimen be inclined so that the exterior angle of incidence is $i_{0}$, it may be shown that $\Delta=\frac{2 t}{\lambda}\left[\mathrm{I}-\sqrt{\mathrm{I}-\frac{2 n \partial n}{\sin ^{2} i_{\mathrm{o}}}}\right]$ in which $n$ is the index of refraction of the glass and $\partial n$ the excess of this index over that of the stria.

For values of $i_{\mathrm{o}}$ not too small $\Delta=\frac{2 t}{\lambda} \frac{n d n}{\sin ^{2} i_{\mathrm{o}}}$

The limiting value for real values of $\Delta$ is given by $\sin ^{2} i_{0}=2 n d n$ and as this is approximately $\sin i=0.05$ the corresponding value of $2 n \partial n$ is 0.0025 whence $\Delta=\frac{2 t}{\lambda} \cdot \frac{0.0025}{\sin ^{2} i_{\mathrm{o}}}$

Taking a moderate value of $i_{\mathrm{o}}$, say, corresponding to $\sin i_{\mathrm{o}}=0.2$ the corresponding observed value of $\Delta$ is 0.45 , which gives $t=18 \lambda$ which corresponds to a thickness of about one one-hundredth of a millimeter.

It results from the foregoing that isolated striae are of no more importance than are the bubbles found in some of the best telescope objectives.

In the case of striae of the second class this is not true in general, so that it is not desirable to attempt to utilize such glass for optical purposes where a high resolving power is required.

Nevertheless, it is important to add that for many purposes, such as for binoculars, gun-sight telescopes, periscopes, etc., such glass may give excellent results if the planes of striation are perpendicular to the line of sight. ${ }^{1}$ Such glass should not be used for prisms.

In this report there is no attempt to account for the presence of striae of either class, but with all due reserve it may be surmised that they are possibly due to different causes, the former (single isolated striae) possibly resulting from material of different

${ }^{1}$ A specimen of glass exceptionally badly striated when viewed end on was placed between collimator and observing telescope of the spectroscope (aperture $2^{\prime \prime}$ ) which gives admirable definition. When the specimen was introduced with the striations perpendicular to the line of sight, no deterioration of the definition was observed. Hence a lens of such glass would perform quite as well as if the striation were not present. 
index (which may be melted from the stirrer itself or from other material which follows the stirrer). The second class of striae are more likely due to variations in the mass of the material and have no immediate connection with the stirrer.

In the absence of knowledge of the technique of glass production it would be presumptuous to hazard advice concerning the possibility of eliminating striae. But it seems clear that original homogeneity of materials and as high a temperature as the material will bear are desirable.

Washington, November, 1918. 